\title{
A clustering-based failure mode and effect analysis model and its application to the edible bird nest industry
}

\author{
Kai Meng Tay $\cdot$ Chian Haur Jong $\cdot$ \\ Chee Peng Lim
}

Received: 24 November 2013 / Accepted: 29 May 2014

(C) The Natural Computing Applications Forum 2014

\begin{abstract}
Failure mode and effect analysis (FMEA) is a popular safety and reliability analysis tool in examining potential failures of products, process, designs, or services, in a wide range of industries. While FMEA is a popular tool, the limitations of the traditional Risk Priority Number (RPN) model in FMEA have been highlighted in the literature. Even though many alternatives to the traditional RPN model have been proposed, there are not many investigations on the use of clustering techniques in FMEA. The main aim of this paper was to examine the use of a new Euclidean distance-based similarity measure and an incremental-learning clustering model, i.e., fuzzy adaptive resonance theory neural network, for similarity analysis and clustering of failure modes in FMEA; therefore, allowing the failure modes to be analyzed, visualized, and clustered. In this paper, the concept of a risk interval encompassing a group of failure modes is investigated. Besides that, a new approach to analyze risk ordering of different failure groups is introduced. These proposed methods are evaluated using a case study related to the edible bird nest industry in Sarawak, Malaysia. In short, the contributions of this paper are threefold: (1) a new Euclidean distance-based similarity measure, (2) a new risk interval measure for a group of failure modes, and (3) a new analysis of risk ordering of different failure groups.
\end{abstract}

K. M. Tay $(\bowtie) \cdot$ C. H. Jong

Faculty of Engineering, Universiti Malaysia Sarawak,

Kota Samarahan, Malaysia

e-mail: tkaimeng@yahoo.com; kmtay@feng.unimas.my

C. P. Lim

Centre for Intelligent Systems Research, Deakin University,

Victoria, Australia
Keywords Failure mode and effect analysis . Fuzzy ART - Similarity measure - Risk interval measure · Risk ordering

\section{Introduction}

Failure mode and effect analysis (FMEA) is a popular and effective problem prevention methodology for defining, identifying, and eliminating potential failures and errors of a system, design, process, or service [1]. A search in the literature reveals that FMEA has been used in a wide variety of application domains, e.g., aerospace [2], automotive [1], nuclear [3], electronic [4], manufacturing [5], chemical [6], mechanical [7], health care and hospital [8$10]$, agriculture [11, 12], and ocean engineering [13, 14]. The main usefulness of FMEA is to identify potential failure modes of a system, understand the causes and effects of each potential failure mode, and determine actions to eliminate or reduce the risk of failure modes [1]. Traditionally, the risk of a failure mode is determined by computing the Risk Priority Number (RPN) [1]. The RPN model considers three factors as its inputs, i.e., severity $(S)$, occurrence $(O)$, and detect $(D)$, and produces an RPN score (i.e., multiplication of $S, O$, and $D$ ) as the output [1]. $S$ and $O$ are seriousness and frequency of a failure mode, respectively, while $D$ is the effectiveness of the existing measures in detecting a failure before the failure effect reaches the customer [1].

Regardless of the popularity of FMEA, the use of the traditional RPN model in FMEA is arguable [2, 15]. In [15], a review of various risk evaluation methods as alternatives to the traditional RPN model was presented. The existing methods are grouped into five categories, i.e., multi-criteria decisionmaking (MCDM) methods, mathematical programming (MP) 